MATEC Web of Conferences 3, 01031 (2013)

DOI: $10.1051 /$ matecconf/20130301031

(C) Owned by the authors, published by EDP Sciences, 2013

\title{
Liquid-liquid equilibria in demixing amines: a thermodynamic approach
}

\author{
K. Ballerat-Busserolles ${ }^{1,2}$, Y. Coulier ${ }^{1,2}$, V. Majer ${ }^{1,2}$, and J-Y. Coxam ${ }^{1,2}$ \\ ${ }^{1}$ Clermont Université, Université Blaise Pascal, Institut de Chimie de Clermont-Ferrand, B.P 80026, F-63171 Aubière, \\ France \\ ${ }^{2}$ CNRS, UMR 6296, ICCF-TIM, B.P 80026, F-63171 Aubière, France
}

\section{Introduction}

Carbon dioxide $\left(\mathrm{CO}_{2}\right)$ Capture from post-combustion industrial effluents will substantially contribute to the reduction of anthropogenic emission of carbon responsible for global warming. The chemical absorption of $\mathrm{CO}_{2}$ in mixed solvents (typically aqueous solution of alkanolamines) is considered as a promising avenue for its capture before transportation and storage [1]. Research projects [2] are developed in order to reduce the energy costs of the regeneration step in processes working on absorption-desorption cycles. $\mathrm{CO}_{2}$ desorption in the stripper requires substantial heating of the whole charged solution flowing out the absorber. A new class of amine, namely the demixing amines may be then valuable for new capture process [3]. These amines show a liquidliquid phase separation at moderately elevated temperatures [4]. As shown on fig. 1, such a phase separation at the output of the absorber unit will concentrate carbon in water rich phase.

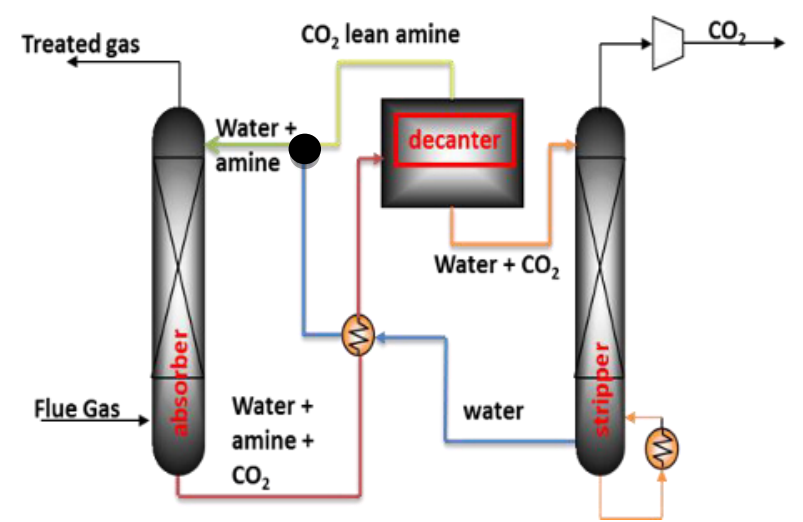

Figure 1. Schematic representation of $\mathrm{CO}_{2}$ separation using demixing amine as chemical absorbent (DMX ${ }^{\mathrm{TM}}$ process [3]).

Then only water rich part of the solvent containing most of the $\mathrm{CO}_{2}$ will be heated in the regeneration step. In order to select possible demixing amines and design new process units it is necessary to analyze the parameters that affect the phase separation.
The presentation will focus on the phase separations occurring in $\{$ water + alkyl piperidine $\}$ binary systems. The phase diagrams $(\mathrm{T}, \mathrm{x})$ are obtained from both calorimetric and visual methods. The amines were chosen to analyze the effects of the position and size of the substituted group. First influence of the $\mathrm{CO}_{2}$ dissolution on the liquid-liquid phase separation will also be reported.

\section{Experimentals}

\subsection{Differential scanning calorimeter}

The liquid-liquid separation temperature can be determined from the detection of the associated energy effect. For this purpose we used microcalorimeters (Setaram microDSC III and $\mu \mathrm{SC}$ ) based on Calvet principle, equipped with $1 \mathrm{~mL}$ cells, and operating in scanning temperature mode from $253 \mathrm{~K}$ to $393 \mathrm{~K}$. The calorimetric signal is the differential thermal flux between the measuring cell filled with the solution and the reference cell filled with water. All experiments are performed at constant pressure and volume with no vapor phase. The liquid-liquid separation temperature corresponds to the first point on thermogram where the thermal flux diverges from baseline. An example of the thermogram obtained with an aqueous solution of $\mathrm{N}$ Methylpiperidine $\left(\mathrm{x}_{\mathrm{a}}=0.2\right)$ and water $(\mathrm{x}=0)$ is presented in figure 2. 


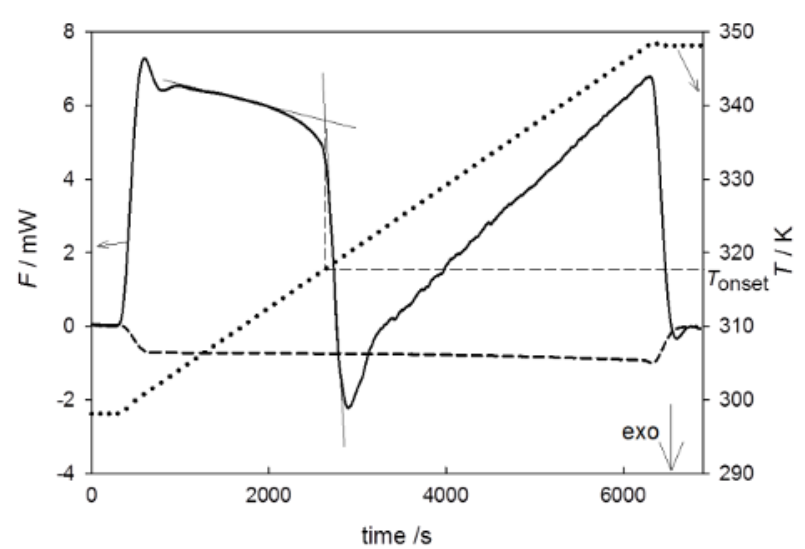

Figure 2. Thermal flux $(F)$ for $\{\mathrm{N}-$ Methylpiperidine + water $\}$ at molar fraction $\mathrm{x}_{\mathrm{a}}=0.2$ (full line) and water (dashed line) versus time ; Temperature profile (dotted line) versus time.

The liquid-liquid separation for \{amine - water $\}$ systems is identified on the thermogram by the weak exothermic peak observed at $318 \mathrm{~K}$. The uncertainty on temperatures estimated from reproducibility tests is about $1 \mathrm{~K}$ while phase separation temperature is graphically determined to better than $0.2 \mathrm{~K}$.

\subsection{Liquid-liquid equilibrium cell}

The second method uses a phase equilibrium cell SPM20 from Thar Technologies providing optical information on the liquid-liquid separation. The binary solution was introduced in the cell, and the solution was observed during scanning of temperature. A high pressure chamber of $15 \mathrm{~mL}$ is filled with the amine-water mixture with no vapor space in order to get rid of vapor-liquid equilibrium. Stirring inside the chamber is insured by a magnetic stirrer driven from the exterior. A camera is installed in front of a sapphire window in order to capture images of the mixture during the experiment.

The experimental protocol consists in steeply increasing temperature by small increments in order to insure temperature homogeneity inside the high pressure chamber. The phase separation is visually determined when clear solution goes through a cloud point. Pictures in figure 3 illustrate the experiments carried out with an aqueous solution of 2-Methylpiperidine $\left(\mathrm{x}_{\mathrm{a}}=0.2\right)$ at three temperatures around cloud point.

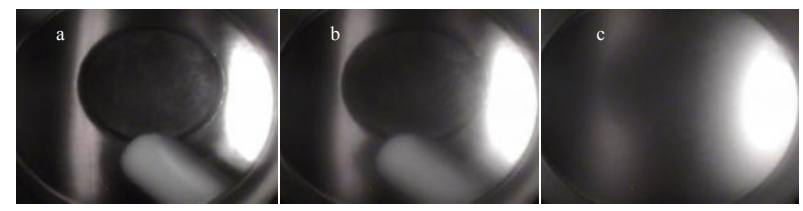

Figure 3. Equilibrium cell pictures for 2-Methylpiperidine + water at molar fraction $x_{a}=0.2$. at $351.3 \mathrm{~K}(\mathrm{a}), 351.5 \mathrm{~K}(\mathrm{~b})$, c: $351.7 \mathrm{~K}(\mathrm{c})$.

Image (a) at temperature $352.3 \mathrm{~K}$ corresponds to transparent monophasic mixture and image (c) at temperature $351.7 \mathrm{~K}$ to a more opaque diphasic mixture. The cloud point is attributed to the situation in image (b) at temperature $351.5 \mathrm{~K}$ where the mixture just starts to become opaque. The uncertainty on temperature of cloud point estimated from reproducibility tests is about $2 \mathrm{~K}$ while uncertainty on such temperature determination for one experiment is better than $0.4 \mathrm{~K}$.

\section{Results}

The liquid-liquid phase diagrams of $\mathrm{N}$-methylpiperidine, 2-methylpiperidine and $\mathrm{N}$-ethylpiperidine in water have been determined using the calorimetric and optical methods. The figure 4 shows the three diagrams obtained.

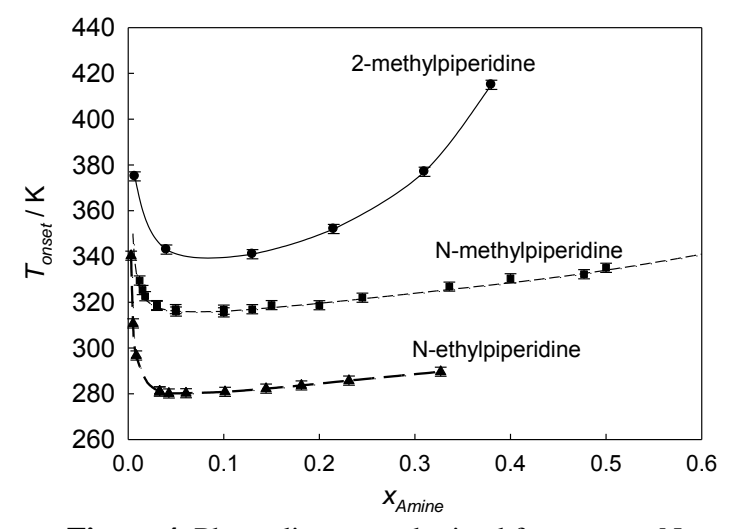

Figure 4. Phase diagrams obtained for water $+\mathrm{N}$ -

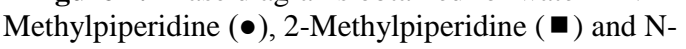
ethylpiperidine ( $\mathbf{\Delta})$.

Our results are consistent with those obtained by Stevenson [5] within the experimental uncertainties for $\mathrm{N}$ - and 2- methylpiperidine, and are systematically lower than those reported by Flaschner et al. [6].

The lower critical solution temperatures (LCST) for the three amines are located at comparable amine molar fractions ( $x_{\mathrm{a}}$ close to 0.07 ). The displacement of methyl group on piperidine cycle from nitrogen to ortho carbon (n- to 2-methylpiperidine) leads to large temperature increase of LCST point ( $315 \mathrm{~K}$ to $339 \mathrm{~K}$ ). The difference in miscibility of water with the two amines can be explained in terms of hydrogen bonding involving free electron pair on nitrogen atom. In the case of $\mathrm{N}$ Methylpiperidine the establishment of hydrogen bond is hindered by the methyl group on nitrogen atom and the miscibility domain observed is then smaller than with 2Methylpiperidine.

Contrarily, the increase of the alkyl length (N-methyl to N-ethyl piperidine) on the nitrogen, increasing the hydrophobicity of the molecule, decreases the temperature of dimixion ( $315 \mathrm{~K}$ to $280 \mathrm{~K}$ ).

The same diagrams were established for systems \{water + methylpiperidine\} saturated with $\mathrm{CO}_{2}$. The phase diagrams obtained are reported in figure 5 . 


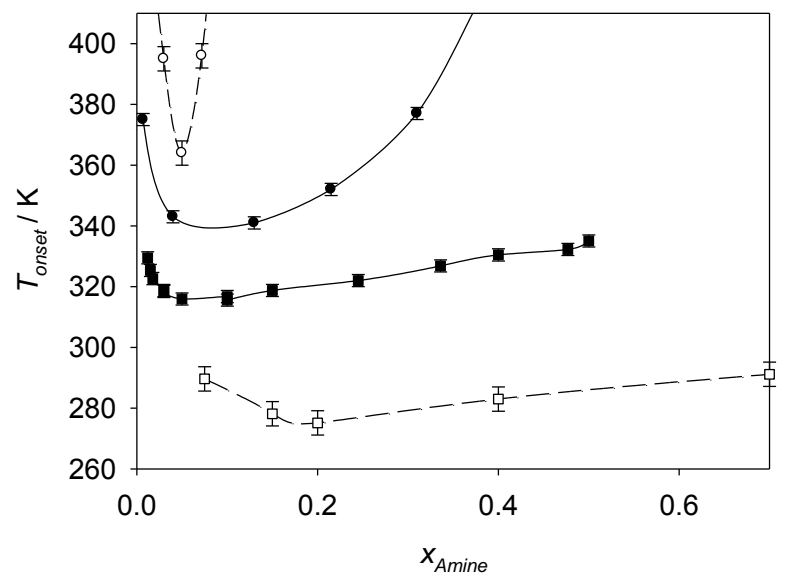

Figure 5. Phase diagrams obtained for water $+\mathrm{N}-$ Methylpiperidine $(\bullet, 0)$, 2-Methylpiperidine $(\boldsymbol{\square}, \square)$ without (full symbols) and with $\mathrm{CO}_{2}$ (open symbol).

The LCST of N-methylpiperidine is clearly moved to the highest molar fractions and the temperature of phase separation is lowered. This may be related to the presence of carbonates in solutions. In the case of 2methylpiperidine, the LCST is slightly displaced to lower molar fractions, and the temperature are increasing, inducing a better stability of the homogeneous solution. In that case, the domain of demixion is very narrow.

\section{Modeling}

The liquid-liquid equilibrium are determined using the representation of the molar gibbs enthalpy of the mixing $\Delta_{\text {mix }} G$ as a function of the molar fraction of amine, as shown in figure 6 .

In the case of a system with liquid-liquid phase separation, $\Delta_{\text {mix }} \mathrm{G}$ exhibits two minimums. The intersection of $\Delta_{\text {mix }} G$ with the common tangents of the two minimums gives the molar fraction of the phases at equilibrium at a given temperature.

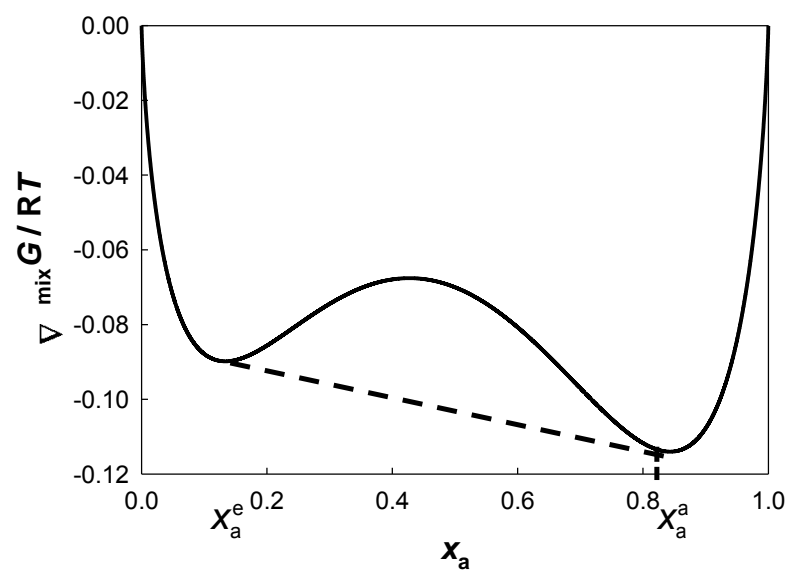

Figure 6. Representation of the molar gibbs enthalpy of the mixing.
The molar gibbs enthalpy $\Delta_{\text {mix }} \mathrm{G}$ of the mixing as a function of the molar fraction of amine is determined as following:

$$
\Delta_{m i x} G=g^{E}+\Delta_{m i x} g^{i d}
$$

The ideal gibbs energy of mixing is defined as

$$
\Delta_{m i x} g^{i d}=R T\left(x_{1} \ln x_{1}+x_{2} \ln x_{2}\right)
$$

while the excess gibbs energy is calculated using UNIQUAC model. In the UNIQUAC model the excess gibbs enthalpy of the component is described by a combinatorial and a residual contribution.

$$
g^{E}=g^{E, c o m b}+g^{E, r e s}
$$

The combinatorial term considers the differences in the size and shape of the molecules expressed as a function of structural parameters, while the residual contribution is related to the molecular interactions in solution. This last contribution is expressed with adjustable parameters, with a quadratic dependency with temperature. This expression leads to the determination of 6 adjustable parameters. The correlation obtained for $\{\mathrm{N}$ methylpiperidine +water $\}$ is shown in figure 7 .

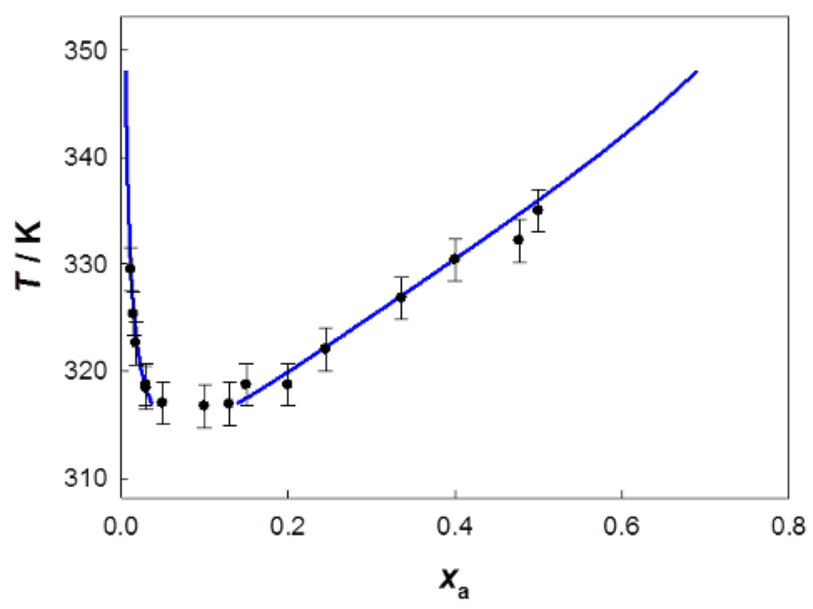

Figure 7. Liquid-liquid phase diagram and UNIQUAC correlation for $\mathrm{N}$-methylpiperidine+water systems.

The model correlates perfectly the experimental curve. However the use of this model is difficult in the region of lower critical solution temperature (LCST) as the two minimums of the gibbs energy of the mixing are too close one from the other.

\section{Conclusion}

The phase diagrams of methyl and ethylpiperidines have been determined using optical and calorimetric technics. It has been shown that the size and position of the substituent influences strongly the temperature of liquidliquid separation as well as the LCST. The presence of $\mathrm{CO}_{2}$ in the solution leads to very different behaviours of the phase diagrams. These differences have to be studied 
for the other substituted piperidines in order to understand the structure-properties of such systems.

\section{Acknowledgements}

The work was started with the financial support of ACACIA DGE (French General Direction for Enterprise), Grand Lyon and AXELERA through the project ACACIA and is actually supported by the French National Agency of Research (ANR) jointly with Natural Sciences and Engineering Research Council of Canada (NSERC), with acronyme DACOOTA.

\section{References}

1. L. Raynal, P-A. Bouillon, A. Gomez, P. Broutin, Chemical Engineering Journal 171742 (2011)

2. L. Rodier, K. Ballerat-Busserolles, J-Y. Coxam, J. Chem. Thermodynamics 42773 (2010)

3. P.-A. Bouillon, M. Jacquin, L. Raynal, IFP.Energies nouvelles, Editor. 2012: France

4. Y. Coulier, K. Ballerat-Busserolles, L. Rodier, J-Y. Coxam, Fluid Phase Equilibria 296206 (2010)

5. R.M. Stephenson, Journal of Chemical \& Engineering Data 38428 (1993)

6. O. Flaschner and B. MacEwen, Journal of the Chemical Society Transactions 931000 (1908) 